\title{
A New High Channel-Count, High Scan-Rate, Data Acquisition System for the NASA Langley Transonic Dynamics Tunnel
}

\author{
Thomas G. Ivanco, ${ }^{1}$ Martin K. Sekula, ${ }^{1}$ and David J. Piatak, ${ }^{2}$ \\ NASA Langley Research Center, Hampton, VA 23681 \\ Scott A. Simmons, ${ }^{3}$ Walter C. Babel, ${ }^{4}$ Jesse G. Collins, ${ }^{5}$ and James M. Ramey ${ }^{5}$ \\ Jacobs Technology, Inc., Hampton, VA 23681 \\ and \\ Dean M. Heald ${ }^{5}$ \\ Analytical Services \& Materials, Inc., Hampton, VA 23681
}

\begin{abstract}
A data acquisition system upgrade project, known as AB-DAS, is underway at the NASA Langley Transonic Dynamics Tunnel. AB-DAS will soon serve as the primary data system and will substantially increase the scan-rate capabilities and analog channel count while maintaining other unique aeroelastic and dynamic test capabilities required of the facility. AB-DAS is configurable, adaptable, and enables buffet and aeroacoustic tests by synchronously scanning all analog channels and recording the high scan-rate time history values for each data quantity. AB-DAS is currently available for use as a stand-alone data system with limited capabilities while development continues. This paper describes AB-DAS, the design methodology, and the current features and capabilities. It also outlines the future work and projected capabilities following completion of the data system upgrade project.
\end{abstract}

\section{Introduction}

$\mathrm{T}$ HE NASA Langley Research Center (LaRC) Transonic Dynamics Tunnel (TDT) has been operational since 1960 investigating a wide range of aeroelastic and non-aeroelastic phenomena. ${ }^{1-5} \mathrm{~A}$ dedicated aeroelastic test facility, the TDT is a large, variable pressure, transonic wind tunnel that can use either air or heavy gas (currently R134a) as a test medium. Unlike typical wind-tunnel testing, which focuses on measuring the aerodynamics experienced by a flight vehicle, the study of aeroelasticity considers the structural response of the flight vehicle when exposed to representative aerodynamics. Typically regarded as the world's premier aeroelastic test facility, ${ }^{6-10}$ TDT fulfills a unique niche in the wind tunnel infrastructure as a result of its unparalleled ability to manipulate fluidstructure scaling parameters.

The TDT is a continuous-flow, single-return, slotted-test-section wind tunnel capable of testing at total pressures from 0.005 to 1.0 atmospheres, over a Mach number range from near zero to 1.20. The test section of the TDT is 16 by 16 feet square with cropped corners yielding a cross-sectional area of 248 square feet. Various model support systems are available offering a wide range of options to enable proper simulation of many dynamic and static phenomena. Models can be mounted with: various sting mounts; various sidewall mounts; a floor-mounted turntable; a two-cable, free-flight support system; a helicopter test apparatus; a tilt-rotor test apparatus; or custom mount systems. ${ }^{1-5,11}$ In addition to the mount type, such as sting or sidewall, various forms of model actuation are also available. The sidewall turntable can either be electrically actuated, or hydraulically actuated with a dynamic system known as the oscillating turntable (OTT). Some tests have also utilized a forced oscillation and/or free-to-

\footnotetext{
${ }^{1}$ Research Aerospace Engineer, Aeroelasticity Branch, Mail Stop 340, AIAA Senior Member.

${ }^{2}$ Research Aerospace Engineer, Aeroelasticity Branch, Mail Stop 340, AIAA Member.

${ }^{3}$ Programmer, Technologies Application Branch, Mail Stop 130.

${ }^{4}$ Programmer, Technologies Application Branch, Mail Stop 130A.

${ }^{5}$ Test Engineer, Subsonic/Transonic Testing Branch, NASA Langley, Mail Stop 340.
} 
pitch cross-bar support in conjunction with the OTT to acquire dynamic stability derivatives. Similarly, the sting can be remotely operated in pitch and roll. Some tests acquire dynamic stability derivatives in pitch or yaw degrees of freedom through the use of a free-to-roll sting mount or dynamic balance installed on a sting.

To support these unique types of aeroelastic and dynamic tests, the TDT possesses a dynamic data acquisition system (DAS) that records time history values of data quantities with synchronous scanning of all analog channels. Steady (static) values are computed as the mean of any data quantity time history. The existing TDT DAS is referred to as the Open Architecture Data Acquisition System or OADAS. OADAS is based upon NEFF 620 signal conditioners/digitizers that are controlled by a MODCOMP computer and has a maximum limit of 256 analog channels. At this maximum channel count, OADAS has a scan rate limitation of $500 \mathrm{~Hz}$. As a result, recent launch vehicle and fixed-wing buffet tests have required the use of supplemental DAS components used in conjunction with OADAS to achieve higher channel counts and scan rates. Additionally, OADAS is aging and the NEFF/MODCOMP hardware is no longer supported by manufacturers. Therefore, an effort was initiated to replace OADAS in order to: increase the scan rate, increase the channel count, increase the reliability, increase user friendliness, and improve upon some features while maintaining synchronous scanning and other unique abilities. This effort has been spearheaded by researchers within the Aeroelasticity Branch (AB) co-located with the TDT. Hence, the new data system has been named AB-DAS. This paper describes AB-DAS development efforts, the current features and capabilities, the design methodology of AB-DAS, and the future development plans and projected capabilities following completion of the TDT DAS upgrade project.

\section{Previous TDT DAS Development Efforts}

Previous DAS development efforts at TDT included multiple supplemental data systems and one long-term OADAS replacement project. The supplemental data systems were used for either an increased scan rate, an increased channel count, or a combination of both.

The first supplemental data system used for recent buffet testing was the Multi-Instrument Integrated Data Acquisition System (MIIDAS) that utilized NEFF 730 hardware. The MIIDAS anti-alias filter was an analog 8-pole Bessel filter coupled with a post-scan digital filter. This system enabled the synchronous scanning of up to 320 analog channels at a maximum scan rate of $12 \mathrm{kHz}$. Use of this system required significant operational support due to limited automation; and lacked many of the basic features of a primary tunnel DAS.

Another supplemental data system was the Piranha III Digital Dynamic Data Monitoring and Analysis System developed by DSPCon. The DSPcon system was capable of scanning up to 288 analog channels at scan rates up to $200 \mathrm{kHz}$. However, only 32 channels were fully conditioned with signal conditioners/amplifiers. Similar to MIIDAS, the DSPcon system required significant operational support, and did not have many of the basic features of a primary tunnel DAS.

Other supplemental systems were temporarily used in TDT and include an aeroacoustic DAS comprised of National Instruments (NI) hardware driven by LabVIEW software, and a rotorcraft DAS also comprised of NI hardware and LabVIEW software.

A long-term TDT DAS upgrade project was initiated by the NASA Aeronautics Test Program personnel and included an exhaustive design study, requirements development, hardware analysis and testing, and input from $\mathrm{AB}$ research personnel. After extensive comparisons and analysis, a hybrid solution proposed by $\mathrm{AB}$ was chosen as the best performing and cost effective hardware. This hybrid solution combined a Precision Filters Inc. (PFI) 28000 series signal conditioner and NI PXIe analog-to-digital converters. The proposed software for the DAS replacement was TestSLATE-based ${ }^{12}$ and required the development of new hardware drivers and many special TDT-required software features. Due to funding constraints and schedule delays, the incorporation of TestSLATE-based software did not fully materialize.

\section{AB-DAS Methodology and Design}

\section{A. Phased Development}

AB-DAS was originally established as a supplemental DAS and funded by a TDT customer project. This customer required faster scan-rate capabilities than OADAS offered. At the time, the TDT DAS upgrade project was underway but could not deliver a functional DAS in time for the customer project; and resurrecting previous and outdated supplemental data systems was also time consuming and costly. Therefore, a proposal was put forth by AB to purchase identical hardware as the TDT DAS upgrade project, develop software in-house to meet the high-speed requirements of the customer's test, and to operate this system in conjunction with OADAS. The new development took on the name "AB-DAS" to differentiate it from the other OADAS replacement effort. Operating as a 
supplemental data system with limited capability, AB-DAS performed well, was reliable, easy to use, and was designed with expansion capability in mind. Follow-on tests requested AB-DAS in lieu of other options, and further capability development continued. As a result of these initial AB-DAS efforts and other funding constraints for the TDT DAS upgrade project, AB-DAS acquired the hardware from the DAS upgrade project and became the new cost-effective replacement option for OADAS following further capability development.

The incremental development of AB-DAS is divided into three phases. Phase I efforts have enabled AB-DAS to serve as a stand-alone data system for TDT for a specific genre of tests that do not require specific features such as a balance task or digital channels. Phase II efforts will be those that increase capability such that AB-DAS can assume all functionality and replace OADAS as the primary tunnel DAS. Phase III efforts will be continued performance enhancements beyond those required to replace OADAS such as the support of thermally compensated steady pressure scanners compatible with new hardware. The phase I development of AB-DAS is complete, and the project is currently in phase II development at the writing of this paper.

\section{B. Hardware Categories}

The hardware components of AB-DAS can be divided into seven categories as illustrated in Fig. 1.

1) Existing tunnel systems (light blue)

2) PSI 8400 steady pressure systems (dark blue), modified existing tunnel systems

3) Signal conditioners (gold)

4) Analog to digital converters (orange), chassis that contain processors for running acquisition software are depicted by a " $P$ "

5) Processing, display, and data storage computer systems (purple)

6) Instrumentation sources (green)

7) Phase II hardware for further capability development (grey)

The analog-to-digital converters consist of NI-PXIe 1085 chassis with embedded 8135 controller/processors. Five such chassis exist and are referred to as: ABNIDASA through ABNIDASE. Chassis ABNIDASA through ABNIDASC contain processors while ABNIDASD and ABNIDASE serve only as expansion chassis for additional cards.

Signal conditioning is accomplished with Precision Filters Inc. (PFI) 28000 series 16-slot chassis with 28124 cards.

Steady pressures are acquired with a Pressure Systems Inc. (PSI) 8400 system. A desktop host computer operates controller software to interface with the PSI 8400 and the other AB-DAS components.

User interface, display, and miscellaneous operations are conducted through a series of desktop workstations named TDTDASWK0 through TDTDASWK3. Similarly, large computations and post-point processing is accomplished on newly acquired high-performance workstations named ABWK1 through ABWK3.

Finally, data throughput and communications is managed with a newly acquired Dell PowerEdge server named ABDAS-SERVER.

\section{General Capabilities}

The maximum channel count of AB-DAS is 512 channels. At this channel count, the DAS can sustain synchronous scan rates of up to $56 \mathrm{kHz}$ for each channel. At reduced channel counts, the sustained scan rate can be increased such that the aggregated rate does not exceed approximately $28,670 \mathrm{kHz}$. For example, with 143 channels or less, a sustained scan rate of $200 \mathrm{kHz}$ per channel is possible. For 71 channels or less, a sustained scan rate of 400 $\mathrm{kHz}$ per channel is possible. The maximum scan rate is $400 \mathrm{kHz}$, regardless of the channel count, with the current acquisition architecture.

Additionally, AB-DAS is capable of acquiring data at different scan-rates at the same time. This is an important feature for tests that need to acquire buffet data with one PXIe chassis while also acquiring aeroacoustic data on a subset of transducers with another PXIe chassis.

\section{Nominal Configurations}

In the nominal configuration, ABNIDASA (daisy-chained to ABNIDASD) will serve as the primary tunnel DAS with 320 analog channels. ABNIDASB will serve as the primary model preparation area (MPA) DAS with 128 analog channels. ABNIDASC will serve as the developmental system with 64 channels. ABNIDASE is reserved for developmental or test-specific expansion needs. 


\section{Optional Configurations}

The analog-to-digital NI-PXIe-6358 cards can be moved in groups of 16 channels as required to support many possible configurations for both the wind tunnel and MPA. For example, ABNIDASD and ABNIDASE can be daisy-chained to ABNIDASA to enable synchronous scanning of all 512 channels.

As required, ABNIDASC can serve as an additional tunnel DAS in conjunction with ABNIDASA when wind tunnel tests (such as the recent Space Launch System buffet tests) require multiple scan rates.

Finally, the option exists to support 256 channels on ABNIDASB for MPA model buildup and checkout if required.

\section{Network Connections}

As shown in the legend of Fig. 1, existing TDT closed-network system (DASnet) connections are depicted by dashed blue lines. DASnet is a communication and control network for all DAS computers and is regulated by bandwidth throttling to limit usage from any one computer. A new storage area network (SAN) was created for data handling of AB-DAS systems, and these connections are depicted by dashed orange lines. Currently the SAN is a 1 Gbit network (single dashed lines) employing CAT 5 cables, and a future capability will include an additional 10 Gbit network (double dashed lines) using CAT 6e cables. The current 1 Gbit network enables rapid transfer of large files since there is no throttling of the network, and it is independent of other DAS communication functions operating on DASnet. The 10 Gbit network intended for phase II development will enable even faster data transfer allowing expansion capability. Additionally, analog instrumentation wiring is depicted by solid green arrows and digital instrumentation wiring is depicted by dashed green arrows. Specialized high-speed back-plane communication protocols, such as PXIe-MC/PCIe/MXI communication, are depicted by solid orange arrows. Finally, the GPIB connections between the PSI 8400 system(s) and host computers are depicted by dashed purple lines.

\section{E. Overview of Data Path Architecture and Communications}

\section{Primary Tunnel Parameters and Time}

The primary tunnel parameters are acquired in the same fashion as that done for OADAS. Total pressure $H$, and test-section static pressure, $P$, are measured through either the primary or secondary Ruska pressure measurement system with digital signals being recorded by an NI PXI/PCI chassis named the "TDT Raw Tunnel Parameter Server." Tunnel total temperature, $T_{o}$, (or stagnation temperature) is recorded at various locations in conjunction with the TDT Heavy Gas system, and the selected gauge (determined by gas operators) is digitally read by TDT Raw Tunnel Parameter Server. Finally, an analog value for gas molar mass fraction, X-gage, of R-134a by comparison to air is also read by the TDT Raw Tunnel Parameter Server. These four quantities: $H, P$, To, and $X$ gage; comprise the primary measurements of tunnel flow. The TDT Raw Tunnel Parameter Server then uses quasireal gas equations detailed in Ref. 13 to generate all of the basic flow property values such as velocity, Mach number, and dynamic pressure. The TDT Raw Tunnel Parameter Server also interfaces with other facility systems such as the Allen Bradley Tunnel drive controller and Heavy Gas control systems. Therefore, the TDT Raw Tunnel Parameter Server, its connections, and all software that resides on it are unchanged and intentionally independent of the other components of the DAS.

AB-DAS conducts a separate primary tunnel parameter calculation on a desktop workstation named "Mach Server." This independent calculation enables the DAS user to specify the molar mass fraction, $X$, in order to stabilize tunnel parameters in the presence of $X$-gage fluctuations. The calculations are conducted by the same software that resides on the TDT Raw Tunnel Parameter Server.

AB-DAS records both sets of tunnel parameters; those coming from Mach Server using $X$, and those coming from TDT Raw Tunnel Parameter Server using $X$-gage. In an effort to save disk space, those tunnel parameters that can be calculated from recorded values, using a widely known equation, were not included. For example, one can easily calculate the speed of sound from either system by simply taking the velocity and dividing by the Mach number.

The primary tunnel parameters from both the TDT Raw Tunnel Parameter Server and the Mach Server are available on DASnet via data socket transfer protocol. This protocol is used to record tunnel parameters during a data acquisition event.

Data system "time" is also provided by the TDT Raw Tunnel Parameter Server. Time information is output by this computer and is utilized by various facility interfaces. Therefore, AB-DAS uses the TDT Raw Tunnel Parameter Server time as the official DAS time on all acquisition engines, data file production, video overlays, and displays in order to provide consistency across all facility systems monitored throughout the TDT complex. Phase II 
development will incorporate the use of a Symmetricon time source synchronized with IRIG-B GPS time that will be available to the various acquisition engines, facility interfaces, and customer systems.

\section{Analog Model and Secondary Tunnel Parameters}

Analog instrumentation on a wind-tunnel model is patched to the data system using appropriate TDT connectors and the patch system located either in the MPA, on the wind-tunnel sting, or the wind-tunnel East wall. Utilizing a mix of TDT patch panel connections and hard-wire connections, these signals are then distributed to the PFI signal conditioners/amplifiers. Details regarding the amplifiers are included in Refs. 14-17. The PFI system is configured

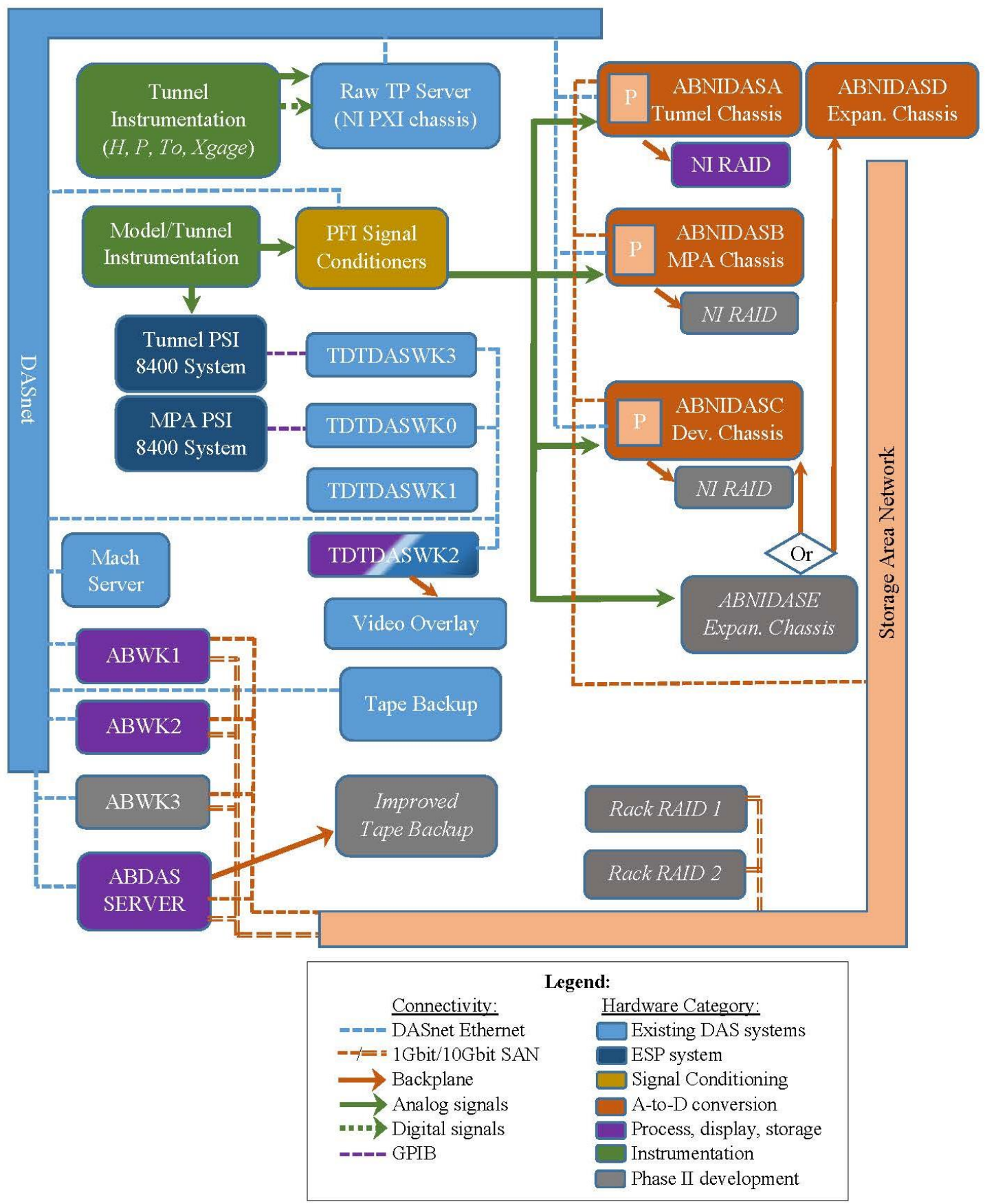

Figure 1. AB-DAS schematic showing connectivity and hardware category. 
through the use of a manufacturer-supplied graphical user interface (GUI) with a software called "LCS." The LCS GUI configures by channel or groups of channels the excitation, gain, filter, auto-balance, and other settings of the amplifier cards. Instructions for the user are provided in appropriate AB-DAS checklists and user guides. A total of eight PFI chassis exist housing a total of 128 4-channel cards providing a total of 512 channels.

The primary output of the PFI signal conditioners is then supplied to the NI-PXIe chassis with PXIe-6358 cards for analog-to-digital conversion. There are five NI-PXIe chassis labeled ABNIDASA thru ABNIDASE. Chassis ABNIDASA, through ABNIDASC contain embedded 8135 PC controllers. These controllers operate using the Windows 7 operating system, and have LabVIEW and MATLAB software installed in order to operate the primary data acquisition engine. ABNIDASD and ABNIDASE are expansion chassis which can be connected via a PXIeMXI-PCI express Daisy-Chain interface. The system is reconfigurable to meet various test-specific requirements and can include the synchronous scanning of all 512 channels through a single processor. Timing for acquisition is accomplished using the embedded $10 \mathrm{MHz}$ chassis clocks, which are synchronized across multiple chassis through the use of NI PXIe-6672 timing cards.

Secondary tunnel parameters, such as vane rotation angle and re-entry flap position, are output from the tunnel PLC controller as analog channels. If analog space permits, these parameters can be scanned synchronously with other analog instrumentation.

Auxiliary outputs are available on the PFI signal conditioners that offer the option of outputting either filtered or wide-band analog data. This auxiliary output can then be used as input to various customer or supplemental data systems, or to the control room for strip chart, frequency analyzer, or other analog monitoring/display or safety-offlight devices.

\section{Tunnel and Model Steady Pressures}

AB-DAS interfaces with a PSI 8400 steady pressure measurement system for the purpose of acquiring multiple steady pressures in conjunction with the dynamic high scan-rate data. The PSI 8400 software driver was recently developed in LabVIEW to support AB-DAS efforts at TDT. The driver software is able to support up to three GPIBconnected PSI 8400 chassis with up to six pressure control units (PCUs) distributed arbitrarily throughout these chassis. Allowable pressure ranges for the PCUs are from 1 to 1015 psi. Likewise, between one and six signal digitizer units (SDUs) can be used in the system and can be arranged to suit the needs of the end user. All nontemperature-compensated scanner types $(2,4,8,16,32,48$, and 64 port scanners) are supported. With these scanners installed, the system has a theoretical (non-averaged, polled) limit of 768 channels at a $10 \mathrm{~Hz}$ scan rate and an upper limit of 6,144 channels at lower scan rates. Maximum tested channels with AB-DAS is on the order of 320 channels at $10 \mathrm{~Hz}$ when scanned in conjunction with dynamic data.

The host software waits for a remote signal to acquire data via a network shared variable in order to begin scanning the 8400 system. This remote signal is generated by the primary data engine on the PXIe chassis. Once the host software begins scanning the 8400 system in accordance with the appropriate setup conditions, the 8400 host computer generates an array containing: channel name, counts, volts, and engineering units (EUs) per channel, and then publishes this data to a network shared variable. The primary data engine on the PXIe chassis records these data values and manages the wind-off tare corrections.

The PSI 8400 host software is able to auto-detect chassis-mounted SDUs and PCUs thereby reducing the opportunity for error. It is also capable of data logging independent of AB-DAS operations. Furthermore, it also has built-in function and calibration checks for the various scanners. Reports are generated for these checks, and the user can specify the allowable limits that generate flags for channels violating the limit.

\section{Near Real-Time Displays and Overlays}

AB-DAS offers near real-time displays of data quantities for monitoring during tests and is compatible with existing facility video overlay systems. To produce data for display while not interfering with high scan-rate acquisition, the PXIe controllers, such as ABNIDASA, continuously acquire data in buffers. The buffer length is configurable by the user, and the default length is a $0.1 \mathrm{sec}$ period. After the acquisition of each buffer, the analog voltage data within the buffer is stored into five different arrays: mean, min, max, one-half peak to peak, and an instantaneous "snap-shot" value from one scan in the buffer. The lengths of these arrays are equal to the number of analog channels, and the value corresponds to the computed voltage quantity for each channel. All five arrays are then broadcast as network shared variables at a rate of $10 \mathrm{~Hz}$. The PXIe controllers also receive tunnel parameter data (via DASnet Ethernet connections and data socket transfer protocol), ESP steady pressure data (via DASnet Ethernet connections and LabVIEW network shared variables), and analog data. Wind-off tare corrections to the ESP data are computed with the NI-PXIe DAS engine and then broadcast as a network shared variable. The DAS engine also broadcasts general information such as: Test, Run, and Point numbers; the take-data signal; the circular- 
archive signal; and DAS time in network shared variables. During system startup or during the load of a new setup file, the PXIe DAS engine also broadcasts user specified equations as a network shared variable.

A TDT control room desktop computer (TDTDASWK2), servers as the master display computer. This computer reads the five analog arrays produced by the DAS controller, the general information read from the setup file, the tunnel parameter values from the TDT Raw Parameter Server and the Mach Server, the tare-corrected ESP values, and the user equations. A master display LabVIEW virtual instrument (VI) then computes all equation values with embedded MATLAB scripts and combines the data with the other sources to form three master arrays of: Data Name, Data Value, and Data Units that are published as network shared variables. These three arrays are then supplied to front-panel displays. Other supplemental facility displays, or customer systems, can read the same LabVIEW network shared variables and are not required to do similar computations, or run MATLAB scripts. This supplemental display software can also operate as a LabVIEW executable so that customer systems do not require LabVIEW in order to display data quantities. Finally, the video overlay VI is also controlled by the master display computer. Utilizing the three data arrays created in the master display VI, the overlay VI publishes near real-time data to the TDT video overlay system.

\section{Data Storage, Backup, and Archive}

AB-DAS has the capacity to acquire incredibly large data files at demonstrated rates in excess of $28 \mathrm{MB}$ per second. This ability requires a creative data flow architecture to realize maximum throughput, and a creative storage and backup architecture to safeguard test data with systems having adequate transfer rates.

As illustrated in Fig. 1, each NI-PXIe data acquisition engine streams raw data files across a backplane interface to an NI-RAID containing approximately $12 \mathrm{~TB}$ of space. These raw data files contain analog channel voltage time histories, ESP pressure time histories, and configuration information captured during the test acquisition period. These data files are identified by a unique Test number, Run number, and Point number to delineate between acquired data sets. After a raw data file is written to the NI-RAID, a data file processing code reads the raw files across the SAN and converts raw data to engineering unit data, applies wind-off-zero offsets, and stores processed data files on an independent processed-file repository RAID. This data storage architecture and methodology enables rapid transfer rates and redundant storage on independent computers.

A multi-tier backup system exists for AB-DAS: tape backup and an independent RAID. First, files from all computers, excluding the data files, are copied to a tape backup system during the night. Secondly, the raw data files from the NI-RAIDs and the processed data files from the repository RAID are copied to a backup RAID during the night. During the day, when no other backup processes are running, all data on the backup RAID are copied to the backup tape system.

Data archive is accomplished as a duty of the TDT DAS manager to archive data post-test using the NASA Langley Central Storage System. Archive is distinct from backup in that it is a long-term storage unrelated to current operations.

Phase II work includes the installation of two rack-mounted 96 TB RAID drives to serve as the primary and backup data repositories. These RAIDs have substantial storage capacity and file transfer rates. Additionally, to enable the backup of larger data files, an upgraded tape backup system will be installed that will communicate directly with ABDAS-SERVER through a serial attached SCSI (SAS) interface enabling larger data transfer rates. ABDAS-SERVER will communicate with other AB-DAS storage systems via the SAN on both 1 Gbit and 10 Gbit networks.

\section{F. Reduced-rate Circular Archive Capability}

The Transonic Dynamics Tunnel was designed to conduct high-risk dynamic testing such as flutter or active controls testing. To mitigate risk to both the model and facility, flow bypass valves are opened to rapidly reduce testsection flow velocity and dynamic pressure. To provide a means of investigating the events prior to, during, and after such an event; a reduced-rate, $1,000 \mathrm{~Hz}$, circular archive capability has been incorporated in AB-DAS for all data quantities. The full-scan rate data is averaged and decimated to a $1,000 \mathrm{~Hz}$ scan rate and stored for up to one minute in a continuous circular (ring) buffer. Anti-aliasing checks are performed for the circular archive data to ensure that the decimation routine adequately averages the input values to avoid aliasing of higher frequency content. Upon bypass valve activation, the pre-event data is recorded to file in addition to post-event data of up to one minute of duration. The option also exists for the user to initiate a circular archive event independent of the bypass valve actuation through a control on the front panel of the operator console. 


\section{G. Unique Wind-off Tare Capabilities}

AB-DAS has multiple wind-off tare capabilities that are user selectable. The option exists for a voltage tare or an engineering unit (EU) tare for analog channels. These tare options allow the user to correctly account for different types of gauge drift. If the cause of the drift is due to a load being applied after calibration (such as the effect of additional model weight on a strain gauge calibration), then an EU tare is preferred. If the gauge drift is likely due to temperature or amplifier drift affecting the zero-load voltage value, then a voltage tare is preferred. Applying the voltage tare is more complex for non-linear gauges and requires the determination of an equivalent zero-load voltage. In practice, minimal differences are noted between the different tare options when using temperaturecompensated instrumentation.

Additionally, AB-DAS offers the opportunity to provide a tare to virtual channels computed by arbitrary userdefined equations. An example where this is especially useful is the utilization of absolute pressure transducers to resolve differential pressure. Absolute pressure transducers do not require a reference pressure line and situations exist where model design may restrict the use of differential transducers. In such a case, the differential pressure can be simulated by subtracting the tunnel static pressure from the measured absolute pressure using a user-defined equation specified in the DAS setup file. During wind-off conditions, the user can then apply an equation tare to zero the value of this equation-derived quantity. The method of application of the equation tare is similar to the EU tare for analog instrumentation.

\section{H. User-Friendly In-Situ Calibration Routine Enabling ANSI Z540.3 Compliance}

AB-DAS has a built-in in-situ calibration routine that enables the user to calibrate any number of analog gauges using a series of standard loads. The user can specify up to a fifth-order polynomial curve fit. The calibration routine automatically produces a report that records all applied loads, the resultant voltages by channel, the coefficients of the calibration fit by channel, and graphs of the calibration fit compared to the applied standard loads for each calibrated transducer. Additionally, temperature and humidity measurements are recorded during calibration, and the test documentation contains unique identifiers for each transducer. If a certified user follows the approved task description and checklist, then the calibration is NIST traceable and ANSI Z540.3 and ISO 9001 compliant.

\section{Data Quality Assurance}

AB-DAS underwent data quality and operational validation checks prior to using the system for a wind-tunnel test. Additionally, AB-DAS has undergone a formal acceptance test and operational readiness review and has since been adopted as an official facility system capable of stand-alone operation within the wind tunnel for a certain genre of tests. A description of some of these tests and checkouts follows.

\section{Basic Functionality Checks}

Basic functionality was tested with the use of a calibrated voltage standard and calibrated signal generator. DC offset values and dynamic signals (sine waves of various frequencies and white noise) were input into the data channels at the sting location. An end-to-end check of each channel was conducted. The magnitude of each DC voltage value accounting for various gain settings was also confirmed. Additionally, phase checks were performed from channel to channel with the use of white noise and discrete frequency signals applied to the calibration bus of the PFI signal conditioners enabling acquisition across all channels. Frequency response functions (FRF), that analyze phase and magnitude as a function of frequency, were conducted from channel to channel to confirm the performance of the amplifiers and analog-to-digital conversion boards. All hardware components performed as predicted by the manufacturer and there was negligible phase shift across all channels for a frequency range of 0 to $100+\mathrm{kHz}$, and all gain values tested (those required for recent testing) performed as expected.

Phase performance was additionally verified by the application of a triangular wave across multiple channels, with the input signal shorted midway during an acquisition record. The occurrence of the short and the peaks of each wave occurred at the same sample in the time history data record for all channels carrying the test signal.

Filter performance was additionally verified by the application of discrete frequency signals well below, slightly below, at, slightly above, well above, and a factor of three above the cutoff frequency. The filters performed as described in the manufacturer's documentation. ${ }^{14-17}$

The Precision Filters Inc. (PFI) 28124 amplifier card uses a 4-pole pulse filter that requires substantial oversampling to get a $20 \mathrm{~dB}$ reduction. There is also a flat filter option, but the output signal is no longer time-domain accurate due to a frequency-dependent phase shift. This behavior is to be expected based upon published curves from the manufacturer, and each filter setting out-performed traditional Butterworth or Bessel filters. 


\section{2. $\quad$ Parallel Testing}

AB-DAS and OADAS were used in parallel during recent wind tunnel tests. This enables data comparisons across both systems, and four specific functions were validated. First, a comparison of the mean values for various channels was conducted throughout testing during both wind-on and wind-off conditions. Second, the higher-order built-in transducer calibration routine of AB-DAS was compared to the results of the linear built-in routine of the OADAS. Third, the time history data were compared between both systems evaluating the dynamic performance. Fourth, the equation function of AB-DAS and the wind-off-zero capability for equation values were validated. Sample data from these validations are presented in this section.

Absolute pressure transducers were used during recent tests and their signals were independently acquired by both data systems; however, the analog signals were conditioned by the same PFI signal conditioner/amplifier prior to digitization. Additionally, tunnel parameters were acquired by both systems during these tests. As an example, Fig. 2 contains a plot of the mean values of an absolute pressure transducer as acquired by both data systems for a multitude of wind-on and wind-off data points. Similarly, Fig. 3 contains a plot of the mean values of sting angle of attack acquired by both systems with a linear fit on OADAS and a polynomial fit on AB-DAS. Finally, Fig. 4 contains a plot of the mean values of tunnel static pressure acquired by both systems for a multitude of data points. As one can see, the mean values are very similar for the pressure transducer and sting angle of attack and nearly identical for the tunnel parameters. This observations lends confidence to the operation of the data system, the loading of the proper coefficients for application to the proper channels, and validates the post-point data file production.

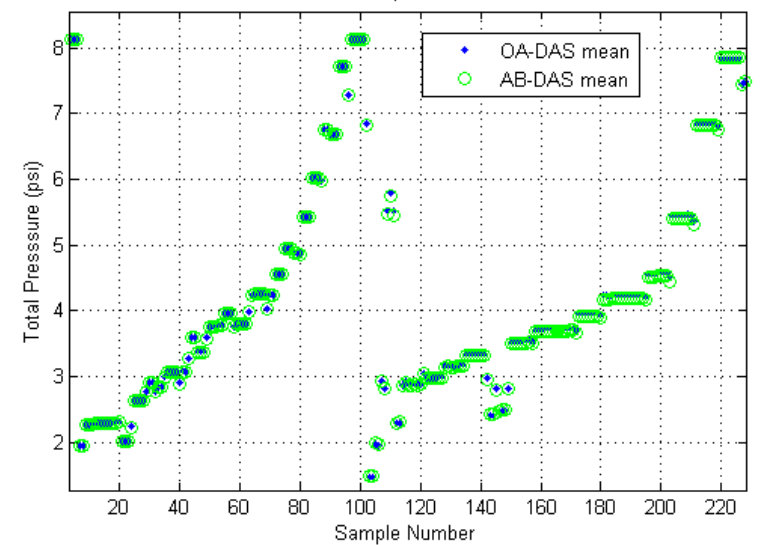

Figure 2. Comparison of data from OADAS and AB-DAS during parallel testing, mean values of an absolute (total) pressure transducer.

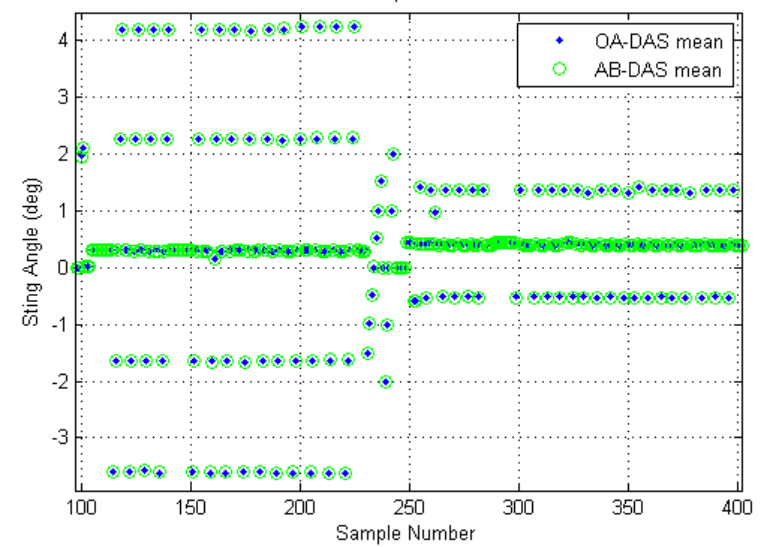

Figure 3. Comparison of data from OADAS and AB-DAS during parallel testing, mean values of sting angle of attack.

Both data systems independently calibrated six absolute pressure transducers with linear calibrations on OADAS (using a built-in function) and polynomial calibrations on AB-DAS (using a built-in function). The mean of the tunnel total, $H$, and static, $P$, pressures, measured by high-precision Ruska absolute pressure sensors, were the calibration standard, therefore subsequent wind-off measurements were employed to verify and compare both calibration methods. The "correct" value of absolute pressure was calculated as the mean value of $H$ and $P$. Error was then calculated for each transducer as the difference between the correct and measured pressure. Figure 5 contains a plot of these error values from both OADAS and AB-DAS data sets. The maximum error in OADAS data is approximately $1.1 \%$ of the transducer's calibrated range ( $7 \mathrm{psi}$ ), whereas the maximum error in the AB-DAS data is an order of magnitude less at $0.11 \%$ of the calibrated range. 


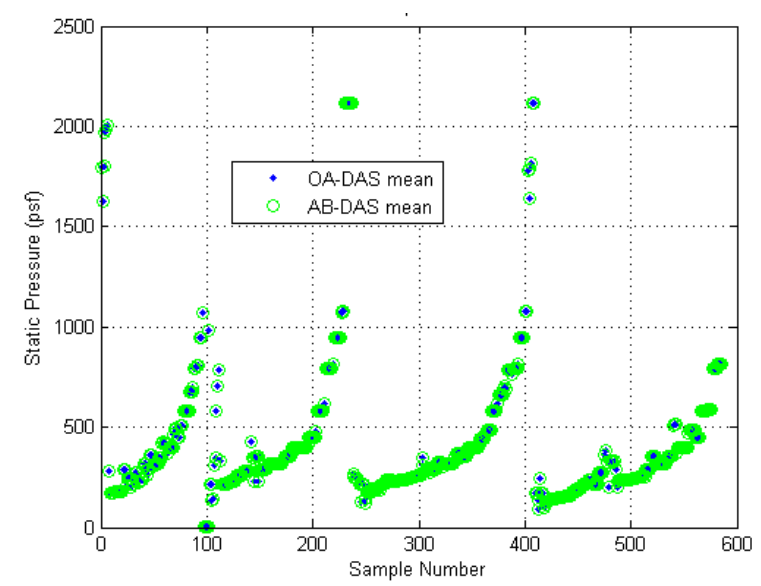

Figure 4. Comparison of data from OADAS and AB-DAS during parallel testing, mean values of tunnel static pressure.

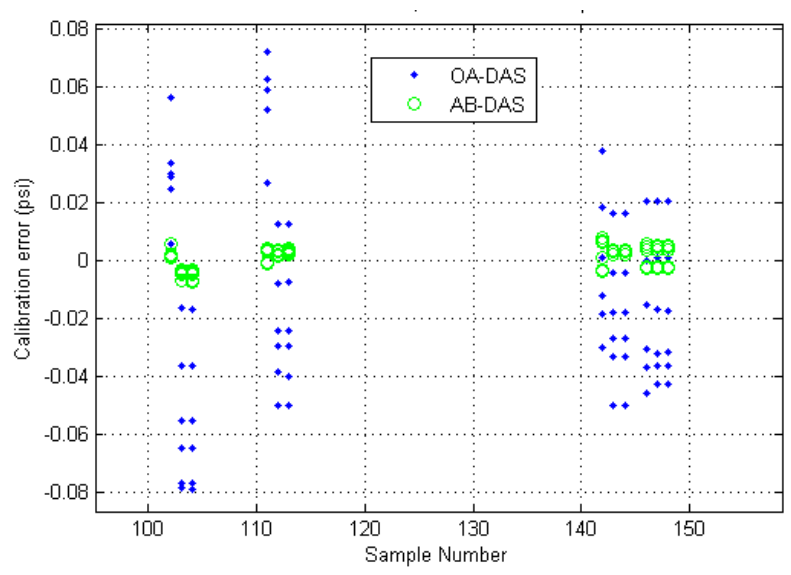

Figure 5. Calibration error values for an absolute pressure transducer, comparison of built-in routine accuracy of OADAS and AB-DAS.

Both OADAS and AB-DAS enable the user to create equations that may contain analog channels, tunnel parameters, and previously calculated quantities. During a recent test, model pitch and roll were determined using a tri-axial q-flex accelerometer package. This package was calibrated in situ with a three-axis angle measurement system (AMS). Additionally, the wind-axis values of model angle of attack (alpha) and side slip (beta) were resolved using Euler rotation angles consistent with TDT procedures. Figure 6 contains a plot of the mean values of model pitch, roll, Alpha, and Beta as recorded by both OADAS and AB-DAS. The similarity of the calculated values validate the arbitrary equation capability of AB-DAS given that these trigonometric relations are rather involved. The results are nearly identical between both systems for most data records; however, there is the occasional outlier (about 5 out of 3804 data records). These outliers where examined, and the differences can be attributed to OADAS acquiring a longer than normal data record (120 seconds) while the sting was in motion. At the same time, AB-DAS was acquiring a standard length data record (30 seconds) and did not capture the same model movements. Therefore, these outliers are not considered as errors of either data system. 

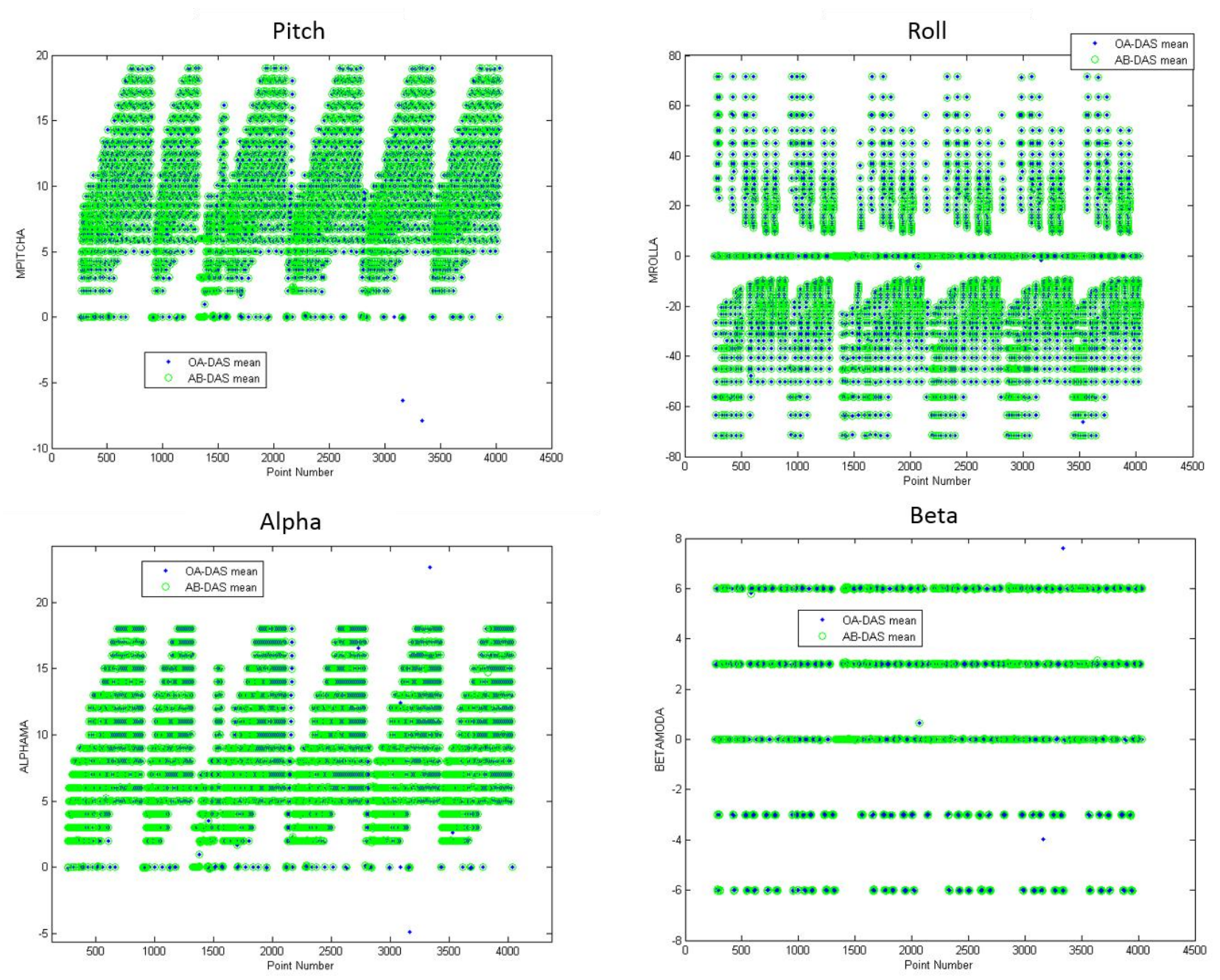

Figure 6. Model pitch, roll (model axis, measured) and alpha, beta (wind axis, calculated from trigonometric relations) degrees; comparison of OADAS and AB-DAS methodology.

\section{Future Work and Planned Capabilities}

Work is ongoing to improve the capabilities of AB-DAS such that it will replace all functionality of OADAS and become the primary data system for the TDT. A summary of the ongoing and future efforts are discussed in this section.

\section{A. Balance Capability}

The most involved and work-intensive process currently underway is the integration of existing MATLAB-based AIAA-standard ${ }^{18}$ and LaRC-standard ${ }^{19}$ wind-tunnel balance routines. When complete, this process will enable the simultaneous use of up to eight balances. The processed balance quantities will include corrections for: balance load interactions, model weight tares at various angles, sting deflection, base pressure, and sting whip. The existing MATLAB routines also translate the measured forces into multiple standard coordinate systems based on the orientation of the balance and test article (model) relative to the flow in the tunnel, as well as automatically calculating aerodynamic coefficients.

\section{B. PSI 8400 Capability Improvement}

The existing PSI 8400 host software incorporates a large number of features that were put in place to simplify future upgrades of the pressure measurement subsystem. The entire PSI 8400 command set has been converted to 
LabVIEW VIs so that follow-on system development can be accomplished more quickly. For example, temperaturecompensated controllers and their associated modules can be used in the system with minimal changes to the existing software. Ethernet communication capability has also been developed with the intent of eliminating the need for GPIB communications. Finally, the existing software was written to leverage command set compatibility of the existing PSI 8400 system with a newer Optimus pressure scanning system.

\section{Other Capability Improvement Efforts}

Ongoing AB-DAS efforts also include the development of a full scan-rate circular archive, an improved DAS log file, improved displays with user-selectable warning and flag levels, and improved phase correlation between master and expansion chassis even though the current capabilities are within requirement standards. Additionally, the development of routines for daily zero, calibration, and phase checks of all signal conditioners and analog-to-digital converters is underway. Furthermore, the capability to acquire digital signals synchronously with the analog data and the ability to output transducer-transducer-logic (TTL) signals is being developed. Finally, the incorporation of IRIG-B GPS time stamps into all data acquisition engines and data files is being developed to enable the post-point correlation of systems with differing scan-rates and with customer-supplied data systems.

\section{Concluding Remarks}

An upgraded data acquisition system, termed AB-DAS, is being developed at the NASA Langley Transonic Dynamics Tunnel and is being implemented in an incremental fashion. An acceptance test has already been completed that enables stand-alone operation of AB-DAS to synchronously acquire high channel-count, high scanrate dynamic data. Furthermore, AB-DAS is capable of acquiring hundreds of steady pressure measurements through the use of a PSI 8400 steady pressure system in conjunction with the high scan-rate analog channels. In its current state, AB-DAS can be used for most TDT tests that do not require a balance or digital channels. The current version of $\mathrm{AB}-\mathrm{DAS}$ has been successfully employed in recent wind-tunnel tests and incorporates many user-friendly features. Some of these features include: unique wind-off tare capabilities; a built-in in-situ calibration routine; compatibility with displays, existing facility systems, and post-point analysis routines; and the ability to broadcast all data quantities to customer systems. The data quality of AB-DAS has been validated through a formal operational readiness review, through the use of calibrated standards, and through parallel testing with the existing tunnel data system. Ongoing efforts will continue to develop additional capabilities to include AIAA-standard and LaRC-standard balance routines. After completion of these efforts, it is projected that AB-DAS will be the primary data system for TDT and will replace the current OADAS system.

\section{References}

${ }^{1}$ Yeager, W. T; Kvaternik, R. G.; "A Historical Overview of Aeroelasticity Branch and Transonic Dynamics Tunnel Contributions to Rotorcraft Technology and Development;” NASA TM-2001-211054; August 2001.

${ }^{2}$ Cole, S. R.; Noll, T. E.; Perry III, B.; "Transonic Dynamics Tunnel Aeroelastic Testing in Support of Aircraft Development;" Journal of Aircraft, Vol. 40, No. 5, September-October 2003.

${ }^{3}$ Cole, S. R.; Keller, D. K.; Piatak, D. J.; "Contributions of the NASA Langley Transonic Dynamics Tunnel to Launch Vehicle and Spacecraft Development;” AIAA Dynamics Specialists Conference, Atlanta, GA, April 2000, AIAA $2000-1772$.

${ }^{4}$ Perry III, B.; Noll, T. E.; Scott, R. C.; "Contributions of the Transonic Dynamics Tunnel to the Testing of Active Control of Aeroelastic Response;" AIAA Dynamic Specialists Conference, Atlanta, GA, April 2000, AIAA 2000-1769.

${ }^{5}$ Ivanco, T. G.; "Unique Testing Capabilities of the NASA Langley Transonic Dynamics Tunnel, an Exercise in Aeroelastic Scaling," AIAA Ground Testing Conference, San Diego, CA; June 2013, AIAA 2013-2625.

${ }^{6}$ Anton, P. S.; Gritton, E. C.; Mesic, R.; Steinberg, P.; Johnson, D.; Block, M.; Brown, M.; Drezner, J.; Dryden, J.; Hamilton, T.; Hogan, T.; Peetz, D.; Raman, R.; Strong, J.; Trimble, W.; "Wind Tunnel and Propulsion Test Facilities, an Assessment of NASA’s Capabilities to Serve National Needs;” The RAND Corporation, Santa Monica, CA; 2004.

${ }^{7}$ Anton, P. S.; Johnson, D. J.; Block, M.; Brown, M.; Drezner, J.; Dryden, J.; Gritton, E. C.; Hamilton, T.; Hogan, T.; Mesic, R.; Peetz, D.; Raman, R.; Steinberg, P.; Strong, J.; Trimble, W.; "Wind Tunnel and Propulsion Test Facilities, Supporting Analyses to an Assessment of NASA's Capabilities to Serve National Needs;" The RAND Corporation, Santa Monica, CA; 2004.

${ }^{8}$ Anton, P. S.; Raman, R.; Osburg, J.; Kallimani, J. G.; “An Update of the Nation's Long-Term Strategic Needs for NASA's Aeronautics Test Facilities;” The RAND Corporation, Santa Monica, CA; 2009.

${ }^{9}$ Under Secretary of Defense (Acquisition, Technology and Logistics); "NASA Aeronautics Facilities Critical to DoD Report to Congress;" Department of Defense, 2007.

${ }^{10}$ Cole, S. R.; Garcia, J. L.; "Past, Present and Future Capabilities of the Langley Transonic Dynamics Tunnel from an Aeroelasticity Perspective;" AIAA Dynamics Specialists Conference, Atlanta, GA; April 2000, AIAA 2000-1767.

${ }^{11}$ Staff of the Aeroelasticity Branch; "The Langley Transonic Dynamics Tunnel;" Langley Working Paper LWP-799, September 1969, reprinted July 1992.

American Institute of Aeronautics and Astronautics 
${ }^{12}$ Www.testslate.com

${ }^{13}$ Kvaternik, R. G.; "Computer Programs for Calculating the Isentropic Flow Properties for Mixtures of R-134a and Air;" NASA Langley Research Center, Hampton, VA, NASA TM-2000-210622, November 2000.

${ }^{14}$ Precision Filters Inc. Staff; 28000-4A-TEST; Operation and Installation of Instructions for the 28000-4A-TEST; Revision D; Precision Filters, Inc.; Ithaca, NY; April 7, 2014.

${ }^{15}$ Precision Filters Inc. Staff; 28124 Card; Operation and Maintenance Instructions; Revision B; Precision Filters Inc.; Ithaca, NY; September 5, 2013.

${ }^{16}$ Precision Filters Inc. Staff; SYSTEM 28000; Operation and Maintenance Instructions; Revision T; Precision Filters Inc.; Ithaca, NY; July 24, 2012.

${ }^{17}$ Precision Filters Inc. Staff; Precision 28124; Quad-Channel Wideband Transducer Conditioner with Voltage and Current Excitation; Specification Sheet; Precision Filters Inc.; Ithaca, NY.

${ }^{18}$ Cahill, D. et. al.; "Recommended Practice, Calibration and Use of Internal Strain-Gage Balances with Application to Wind Tunnel Testing," AIAA R-091-2003, September 2003.

${ }^{19}$ Mercer, C. E., Berrier, B. L., Capone, F. J., Grayston, A. M.; "Data Reduction Formulas for the 16-Foot Transonic Tunnel NASA Langley Research Center," NASA TM 107646, July 1992. 